\begin{tabular}{|c|l|}
\hline Title & Scattering of traveling spots in dissi pative systems \\
\hline Author(s) & Nishiura, Y asumasa; Teramoto, Takashi; U eda, Kei-Ichi \\
\hline Citation & $\begin{array}{l}\text { Chaos: An Interdisciplinary Journal of Nonlinear Science, 15, 047509 } \\
\text { https:/doi.org/10.1063/1.2087127 }\end{array}$ \\
\hline Issue Date & 2005-12 \\
\hline Doc URL & http://hdl.handle.net/2115/6125 \\
\hline Rights & Copyright $\odot 2005$ A merican Institute of Physics \\
\hline Type & article \\
\hline File Information & Chaos15.pdf \\
\hline
\end{tabular}

Instructions for use 


\title{
Scattering of traveling spots in dissipative systems
}

\author{
Yasumasa Nishiura \\ Research Institute for Electronic Science, Hokkaido University, Kita-ku, Sapporo, \\ Hokkaido 060-0812, Japan \\ Takashi Teramoto \\ Chitose Institute of Science and Technology, Chitose 066-8655, Japan \\ Kei-Ichi Ueda \\ Research Institute for Mathematical Sciences, Kyoto University, Kyoto 606-8502, Japan
}

(Received 10 July 2005; accepted 5 September 2005; published online 30 December 2005)

One of the fundamental questions for self-organization in pattern formation is how spatial periodic structure is spontaneously formed starting from a localized fluctuation. It is known in dissipative systems that splitting dynamics is one of the driving forces to create many particle-like patterns from a single seed. On the way to final state there occur many collisions among them and its scattering manner is crucial to predict whether periodic structure is realized or not. We focus on the colliding dynamics of traveling spots arising in a three-component system and study how the transition of scattering dynamics is brought about. It has been clarified that hidden unstable patterns called "scattors" and their stable and unstable manifolds direct the traffic flow of orbits before and after collisions. The collision process in general can be decomposed into several steps and each step is controlled by such a scattor, in other words, a network among scattors forms the backbone for scattering dynamics. A variety of input-output relations comes from the complexity of the network as well as high Morse indices of the scattor. The change of transition manners is caused by the switching of the network from one structure to another, and such a change is caused by the singularities of scattors. We illustrate a typical example of the change of transition caused by the destabilization of the scattor. A new instability of the scattor brings a new destination for the orbit resulting in a new input-output relation, for instance, Hopf instability for the scattor of peanut type brings an annihilation. (c) 2005 American Institute of Physics. [DOI: 10.1063/1.2087127]

One of the fundamental questions for self-organization in pattern formation is how spatial periodic structure is spontaneously formed starting from a localized fluctuation. Two things seem to be necessary to have a periodic pattern starting from a localized seed, one is an intrinsic instability of a seed solution like self-replication, the other is to arrange many localized patterns in order to form a regular periodic pattern through interaction. When the seed solution is a two-dimensional traveling spot, then the interaction occurs mainly through collisions besides weak and long-range interaction. To understand the collisional process is a challenge not only because of its large deformation but also its influence over the global coherent dynamics, for instance, if annihilation occurs upon collision, there is much less chance to have a regular periodic pattern. What is discussed here is to clarify the underlying mechanism to control the scattering dynamics among traveling spots arising in threecomponent reaction diffusion systems. It turns out that hidden saddles called scattors make a traffic control of orbits during the collision process, in fact the orbital behaviors are guided by the stable and unstable manifolds of such scattors, and the output can be classified by looking at the outcome from the scattor. We also discuss how the structural change of the network among scattors influences the input-output relations of scattering dynamics. Such a change is typically caused by a new instability of the scattor, which eventually leads to a drastic change of output after collision.

\section{INTRODUCTION}

Reaction diffusion media have a variety of dynamic response to spatially localized perturbation. If the medium is unstable to inhomogeneous perturbation, then small random fluctuation may cause formation of a periodic structure via Turing instability, however if the medium is locally stable, then perturbation of finite amplitude is necessary. It is well known for excitable systems (monostable media) like FitzHugh-Nagumo equations that localized perturbation exceeding the threshold creates counter-propagating traveling pulses in one dimension (1D) however it does not form a spatially periodic structure on an extended medium and annihilation occurs when they collide. Two things seem to be necessary to have a periodic pattern starting from a localized seed, one is an intrinsic instability of a seed solution like self-replication, the other is to arrange many localized patterns in order to form a regular pattern through interactions. Nonannihilation property becomes important for the latter purpose.

One of the recent remarkable discoveries ${ }^{1-4}$ is a variety of particle-like patterns satisfying the above two requirements, namely they replicate by themselves and behave like 


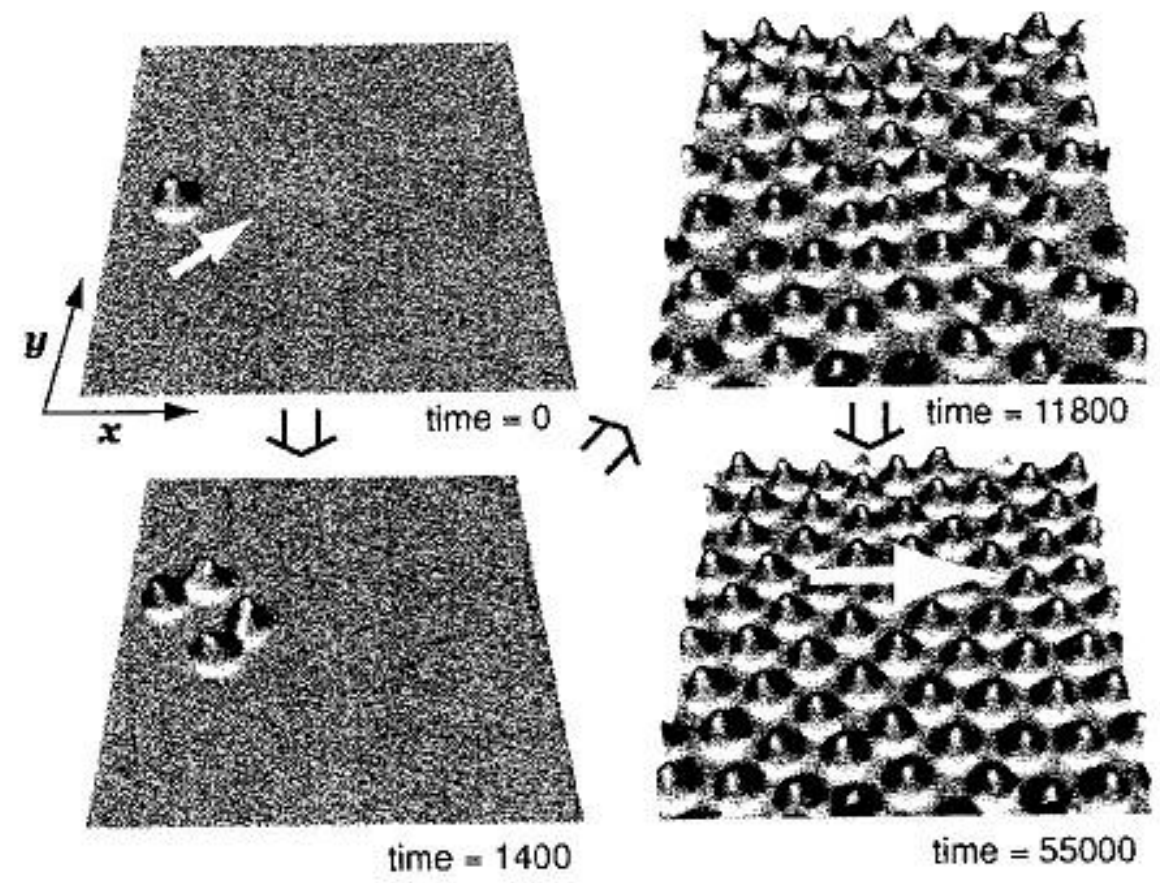

FIG. 1. From a single spot to traveling coherent structure when $\left(f_{0}, f_{1}, \tau\right)$ $=(0.050,0.060,40.0)$ for the model equation (1) with periodic boundary condition. Only $v$ component is shown here.

elastic objects upon collision or merging into a single spot without annihilation. One example is shown in Fig. 1 which solves the model equation (1) in Sec. II: Starting from a single spot, self-replication occurs and the newly born spots travel, collide and eventually settle down to a marching periodic pattern with fixed velocity as in Fig. $1(t=55000)$. The final ordered pattern is maintained dynamically through the interaction with neighboring spots, in fact if one picks up a spot from the final state and sets it in a free space, then it proliferates again, therefore the crowdedness prevents the spots from self-replication and keeps the coherent structure.

Particle-like patterns have been observed experimentally and numerically, for instance, in gas-discharge systems, ${ }^{5-7}$ CO-oxidization process, ${ }^{8-10}$ chemical reactions, ${ }^{1-4,11-14}$ morphogenesis, ${ }^{15}$ and reaction-diffusion systems with a global feedback system. ${ }^{16.17}$ Suppose there are spatially localized moving patterns such as pulses or spots in a free space. A qualitative change of the pattern may occur either by means of interaction with other patterns through collision or intrinsic instability such as splitting or destruction by itself. It is known that if a localized pattern has an intrinsic instability like self-replication, then combining with selfdestruction or annihilation process, it produces in general a complex dynamics like spatiotemporal chaos ${ }^{18}$ or Sierpinski gaskets. ${ }^{19}$ In order to understand the whole dynamics of such complex patterns, a computer-aided geometric approach is quite useful as was shown by Refs. 18,20, and 21 . Note that annihilation does not occur in Fig. 1, so it tends to an ordered state asymptotically. This indicates that understanding the local collisional process is a key to predict the whole dynamics, however the underlying mechanism to control such scattering dynamics is very little known compared with the weak interaction regime ${ }^{11,12}$ partly because it contains a large deformation in infinite dimensional space. A new viewpoint has been presented in Refs. $22-24$, to shed light on this issue, namely hidden saddles called "scattors" make a traffic control of orbits during the collision process, especially the above concept is useful to understand the transition of collision manner, for instance, from bouncing of two traveling spots to annihilating each other. In fact the orbital behaviors are guided by the stable and unstable manifolds of such scattors, and the output can be classified by looking at the outcome from the scattor. There are several related works pointing out the importance of such saddles, see for instance, Refs. 25-28.

The aim of this paper is twofold: First we extensively study the head-on collisions of two-dimensional (2D) traveling spots for the three-component system (1) in Sec. II and find scattors and their interrelations from a global bifurcational viewpoint. Second we discuss how the change of network structure of scattors influences the scattering dynamics. When a scattor undergoes a new instability, then it has a new destination along it, which adds a new part to the existing network and the orbit takes a different route as parameters vary resulting in a new output after collision.

\section{MODEL}

We employ the following three-component reaction diffusion system in order to investigate the scattering dynamics,

$$
\begin{aligned}
& u_{t}=D_{u} \Delta u-\frac{u v^{2}}{1+f_{2} w}+f_{0}(1-u), \\
& v_{t}=D_{v} \Delta v+\frac{u v^{2}}{1+f_{2} w}-\left(f_{0}+f_{1}\right) v, \\
& \pi w_{t}=D_{w} \Delta w+f_{3}(v-w),
\end{aligned}
$$

where $f_{0}, f_{1}, f_{2}$, and $f_{3}$ are all positive parameters related to inflow and removal rates of $u$ and $v$, and inhibition and decay rates for the inhibitor $w$. This can be regarded as an extended system of the Gray-Scott model in the sense that an inhibitor $w$ is added to the two-component Gray-Scott model (2),

$$
\begin{aligned}
& u_{t}=D_{u} \Delta u-u v^{2}+f_{0}(1-u), \\
& v_{t}=D_{v} \Delta v+u v^{2}-\left(f_{0}+f_{1}\right) v .
\end{aligned}
$$

The system (1) is called an activator-substrate-depleted model (Ref. 15). In fact the dynamics of the kinetic part of (1) behaves in a similar way to that of (2) as in Fig. 2. In the sequel we work in the dark-gray region of Fig. 2, i.e., monostable regime. The reason why we employ the above system is that (1) is a representative model which shows most of the interesting scattering dynamics and transitions among them in two-parameter space as well as drift bifurcation as in Fig. 3. Our approach can be applicable to other model systems, especially the concept of scattor in Sec. IV seems to work universally.

All the computations that follow for (1) are done in the following setting: the system size is $4 \times 2$ with the Neumann boundary condition. The grid sizes are $\Delta x=\Delta y=2^{-6}, \Delta t$ $=0.10$ and the five-point difference approximation is employed for the Laplacian. The parameters are set to $\left(D_{u}, D_{v}, D_{w}\right)=\left(2.0 \times 10^{-4}, 1.0 \times 10^{-4}, 5.0 \times 10^{-4}\right)$ and $\left(f_{0}, f_{2}, f_{3}\right)=(0.05,0.50,0.20)$. Spectral computations for eigenvalues and eigenfunctions throughout the paper are done with $64 \times 64$ grids. For convenience, only profiles of the $v$ component are shown throughout the figures. 


\section{B. Three different outputs: repulsion (RE), fusion + drift (FD), and annihilation (AN)}

Let us classify the head-on collision by its output. There are three qualitatively different outputs for (1): repulsion (RE), fusion-drift (FD), and annihilation (AN) depending on two parameters $f_{1}$ and $\tau$ as in Fig. 3 .

Since the standing spots in the light-gray region of Fig. 3 repel each other, the collision process for traveling spots near the drift bifurcation line is expected to inherit its nature, namely they interact weakly and bounce off with keeping their original shapes. This is the case for our system except near the intersecting point $\mathrm{DH}$ of the drift bifurcation curve with the Hopf line in Fig. 3 (i.e., codim 2 point). It is known that there appears an annihilation regime near such a singularity even though the parameter is arbitrarily close to the drift line (note the tip of the AN regime in Fig. 3), in fact we need another singularity, i.e., saddle-node point for this, however we leave the discussions to Ref. 30 . The RE regime expands in the left-up direction. As the velocity of the spot is increased, the distance between two spots at collision becomes shorter, and eventually the dynamics is switched to the FD regime, namely two spots merge into one spot and start to drift in one direction as in Fig. 5(a). The FD regime expands as $\tau$ is increased until the splitting instability emerges. There is another scattering regime in the right-up region of Fig. 3, i.e., AN regime in which two spots collide and annihilate each other. There are two ways of annihilation, fusion type and nonfusion type. Along the left boundary of the AN regime, two spots merge into one body and then annihilate, on the other hand, along the bottom boundary except near the triple junction (TJ), two spots do not merge and they disappear, keeping some distance and these two boundaries converge to $\mathrm{TJ}\left[\left(f_{1}, \tau\right) \approx(0.0636,73.2)\right]$. These observations suggest that there are two categories of scattering dynamics, fusion and nonfusion types. These two cases coexist in the $\mathrm{RE}$ and $\mathrm{AN}$ regimes where nonfusion manner gradually changes into the fusion one as $f_{1}$ is decreased. In this paper we focus only on the fusion case and study the origin of how the tip of the annihilation regime appears near TJ from the global bifurcation viewpoint.

Since $f_{1}$ is the decay rate of the activator $v$, large $f_{1}$ implies no patterns (ground state) and small $f_{1}$ fosters the growth of patterns. On the other hand, $\tau$ represents how quickly the inhibitor $w$ diffuses and responds to the change of the activator $v$, namely, for smaller $\tau, w$ can block the propagation of $v$ and support standing spots, however for larger values, the inhibitor $w$ remains near the colliding area due to the slow response which enhances annihilation.

\section{SCATTORS IN TWO-DIMENSIONAL SPACE}

In order to understand the transitions among RE, FD, and AN regimes, we first need to study the orbital behaviors carefully as parameters cross such boundaries.

An ideal head-on collision has $D_{2}$ symmetry in space, i.e., invariant under left-right and up-down reflections with respect to the collision line, which suppresses translational instabilities. A merging spot in the FD region therefore is supposed to stand still after collision, however, as we will

\section{(a)}

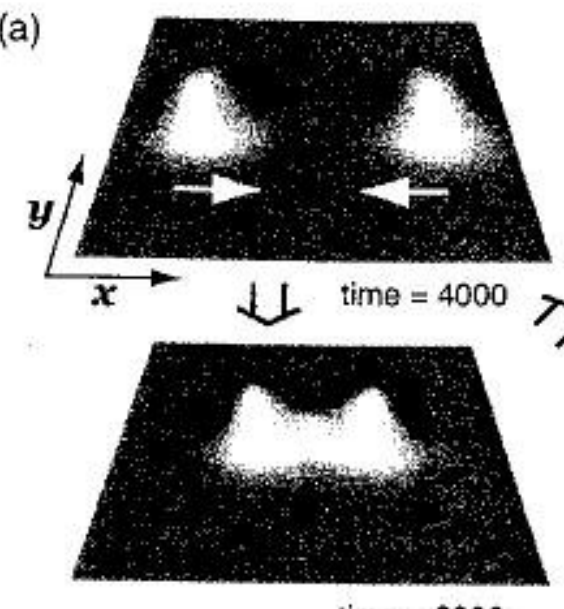

time $=6000$
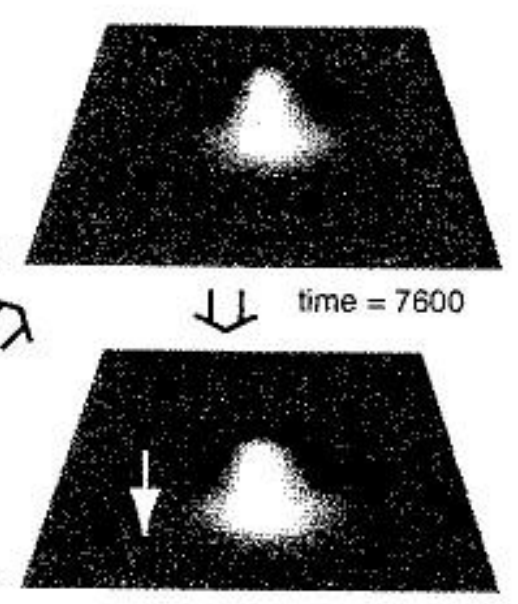

(b)
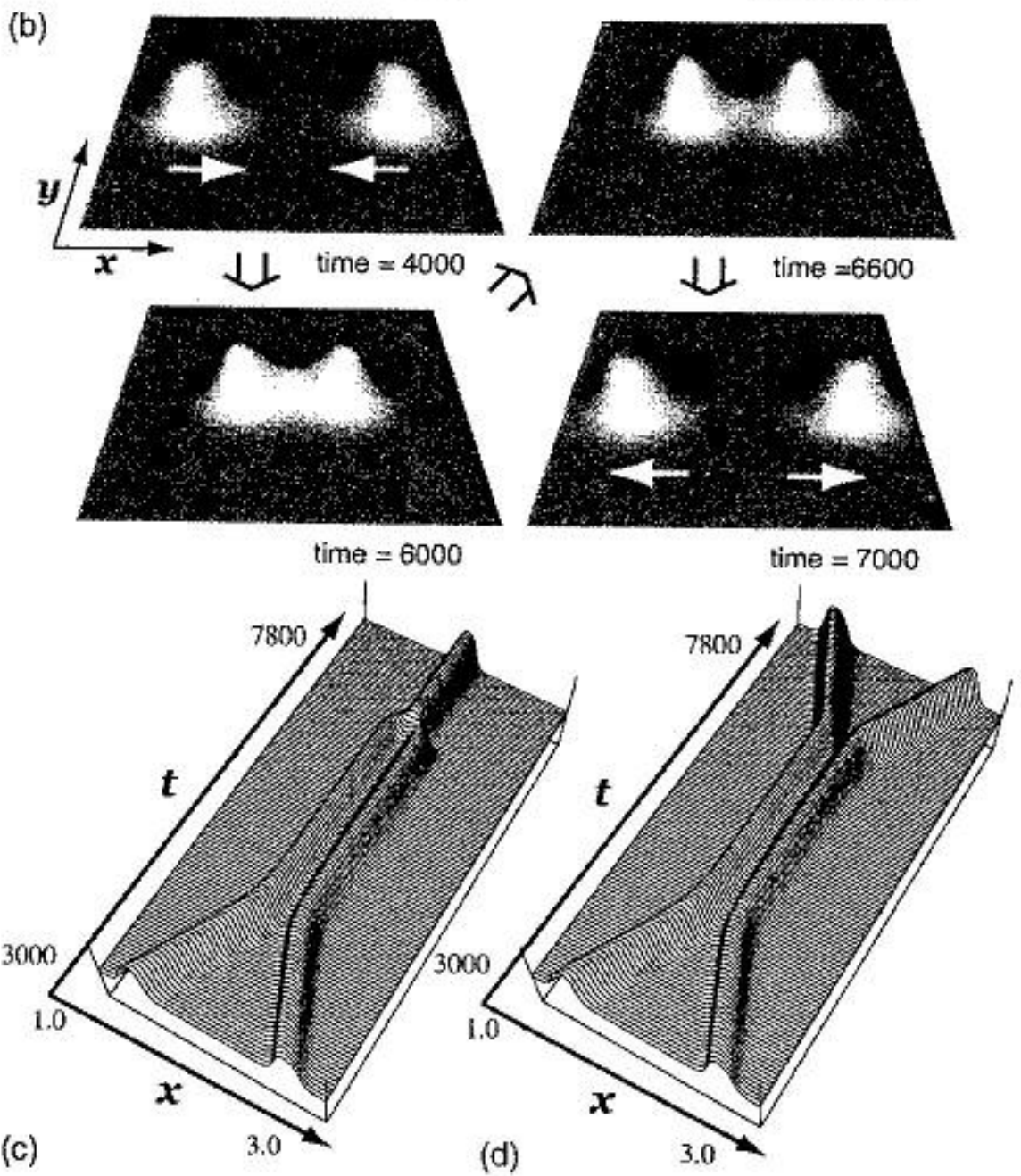

time $=16600$

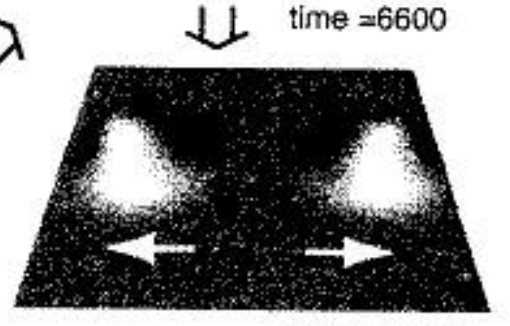

(d)

FIG. 5. Transition from FD to RE at $\tau \approx 63.777$ as $\tau$ is decreased with $f_{1}$ $=0.0625$. (a) Fusion+drift side. (b) Repulsion side. Peanut-shape is clearly visible for both cases, then the middle part of it grows and becomes one body (respectively, decays and splits into two spots) for (a) [respectively, (b)]. The associated time evolutions of the cross section along the $x$-axis are shown in (c) and (d). The orbit stays longer near the peanut shape when the parameters are closer to the transition point.

see in the following simulations, tiny fluctuations or numerical errors actually cause the growth of drifting instabilities after a long time.

\section{A. From repulsion to fusion +drift}

Let us take a closer look at the transition from RE to FD at $\mathrm{I}=\left(f_{1}, \tau\right) \approx(0.0625,63.777)$ in Fig. 3. The orbital behavior on the RE (respectively, FD) side is given by Fig. 5(b) [respectively, Fig. 5(a)]. Both orbits take the peanut shape after collision and stay there for a certain time, then either it splits or two humps fuse into one spot and drift away. Closer to the boundary of two regimes, a longer stay near the peanut shape indicates the existence of a steady state of saddle type, in fact it is numerically confirmed by the Newton method that there exists an unstable steady state of codim 3 the profile of which is very close to that of $t=6000$ in Fig. 5. Two of them 


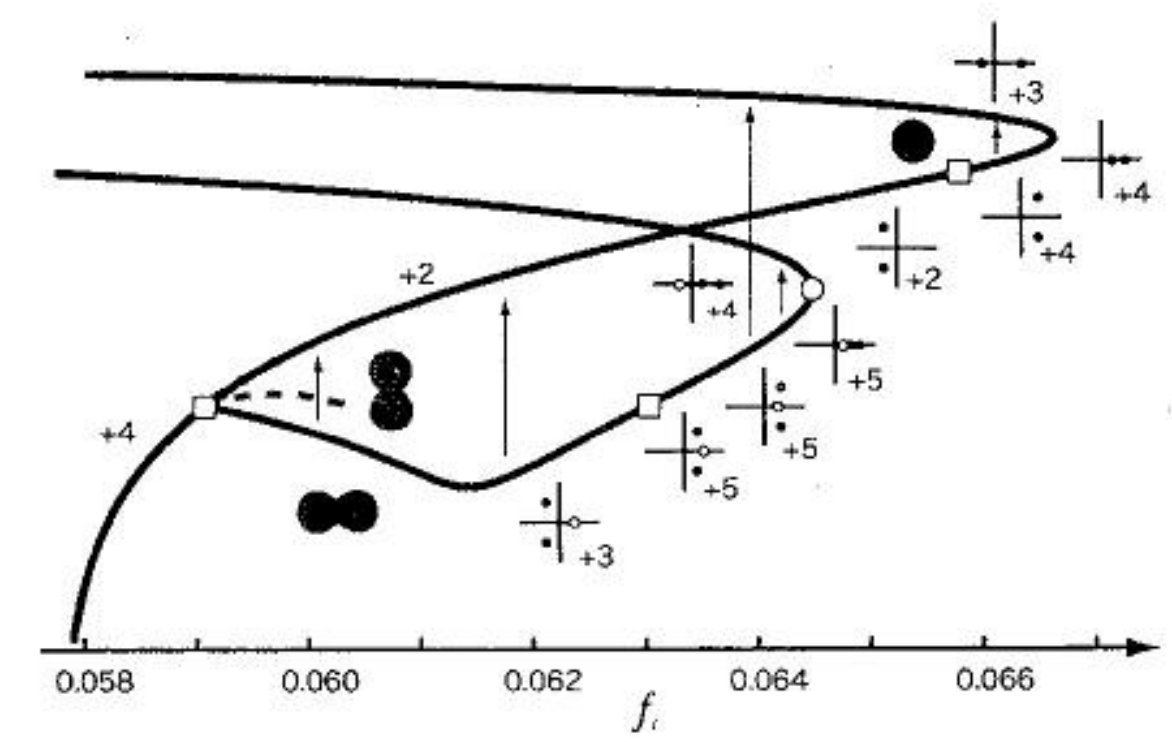

FIG. 6. Global bifurcation diagram for scattors. The bifurcation parameter is $f_{1}$ with $\tau$ being fixed as ( $\approx 63.777$ ). The vertical axis corresponds to the $L^{2}$ norm of the $u$ component. The peanut branch emanates from the disk branch by a $D_{x} \rightarrow D_{2}$ symmetry-breaking bifurcation at $f_{1} \approx 0.0590$. Behavior of eigenvalues are also depicted along the branches. New pairs of complex eigenvalues appear and cross the imaginary axis before the limiting points. The orbital flows near the steady states are indicated by arrows. The Hopf bifurcation point of the peanut branch (respectively. disk branch) is located at $f_{1} \approx 0.0630$ (respectively, 0.0658 ). The parameter $\tau$ does not affect the existence of steady states, hence all the branches persist except their stabilities as $\tau$ is yaried. The number attached to each branch indicates that of unstable eigenvalues for $\tau=76.643$. The Hopf bifurcation point (the broken line in Fig. 3) on the branch of standing spots (large disk) is almost independent of $\tau$, as is the Hopf point on the peanut scattor.

are related to the drift instabilities, however they are not important in our setting due to the symmetry of head-on collisions. There is one more real positive eigenvalue responsible for the symmetry-breaking bifurcation from the disk pattern. In fact, by using a path-tracking software based on AUTO (Ref. 31), a peanut-solution branch can be continued globally with respect to $f_{1}$ and it emanates from the large disk pattern as in Fig. 6. The computation of Fig. 6 is done in a quadrant (i.e., $32 \times 32$ ) of the full solution thanks to the symmetry.

It turns out that the orbits are sorted out along the unstable manifold associated with this real eigenvalue, in fact, by adding two types of perturbation to the peanut pattern, the outputs are consistent with the previous evolutional results as in Fig. 7. More precisely, depending on the sign of $\epsilon$ for the perturbation term $\epsilon \Psi_{1}$, it either grows in the middle of the peanut and becomes a one spot or decays and pinches off into two spots as shown in Fig. 7 where $\Psi_{1}$ stands for the eigenfunction associated with the above real eigenvalue [see Fig. 7(a) ]. Again by the Newton method, it is confirmed that there exists a standing spot at this parameter value close to the profile of $t=7600$ in Fig. 5(a) and it has only drift instability. It eventually therefore starts to move in one direction due to small fluctuation. Note that the drifting direction should not be oblique to the colliding direction as in Fig. 5 due to the symmetry. For later use we call the above two unstable steady patterns "peanut scattor" and "large disk scattor," respectively, which actually sort out the orbits along their unstable manifolds.

\section{B. From fusion+drift to annihilation}

Next we consider the transition between FD and $\mathrm{AN}$ at $\mathrm{II}=\left(f_{1}, \tau\right) \approx(0.064577,90.0)$ in Fig. 3 . The orbits corre-
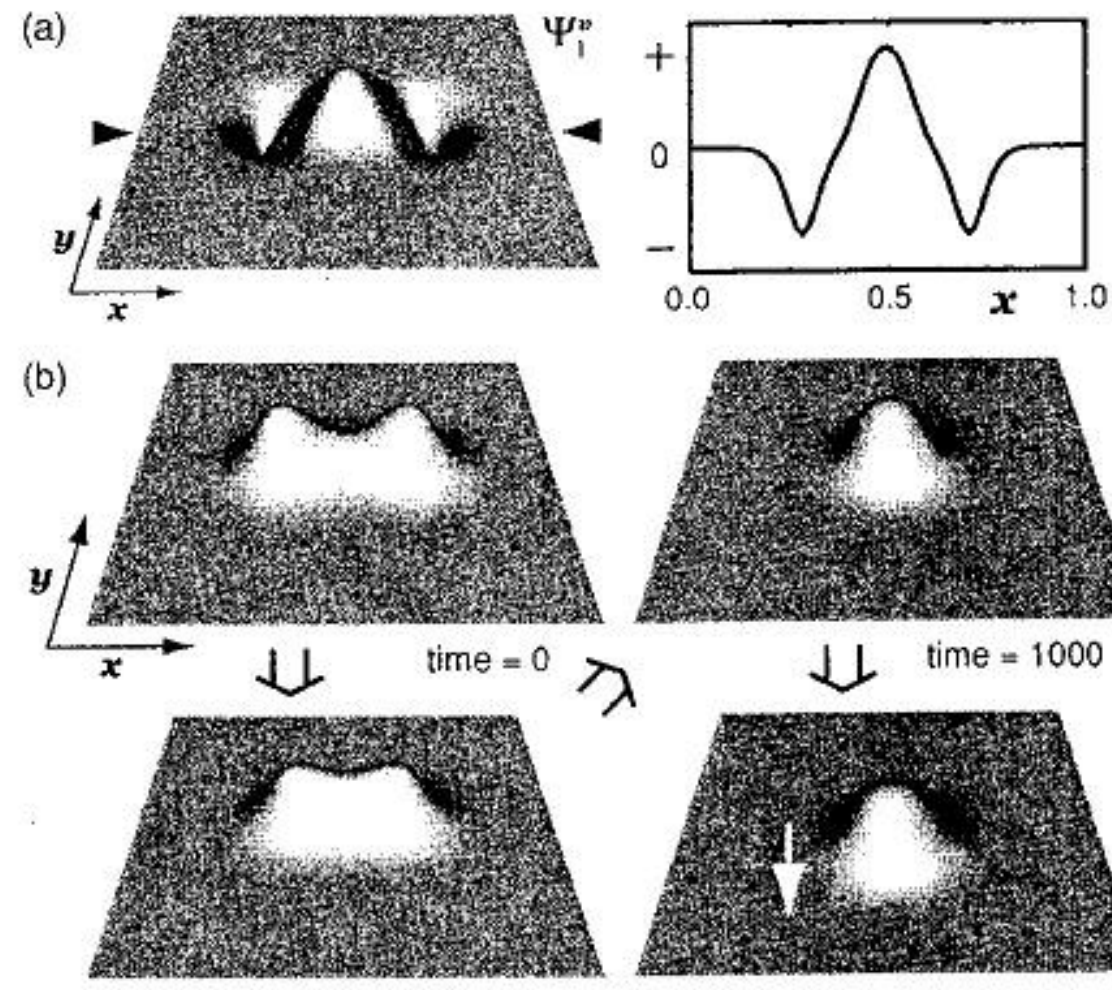

time $=300$
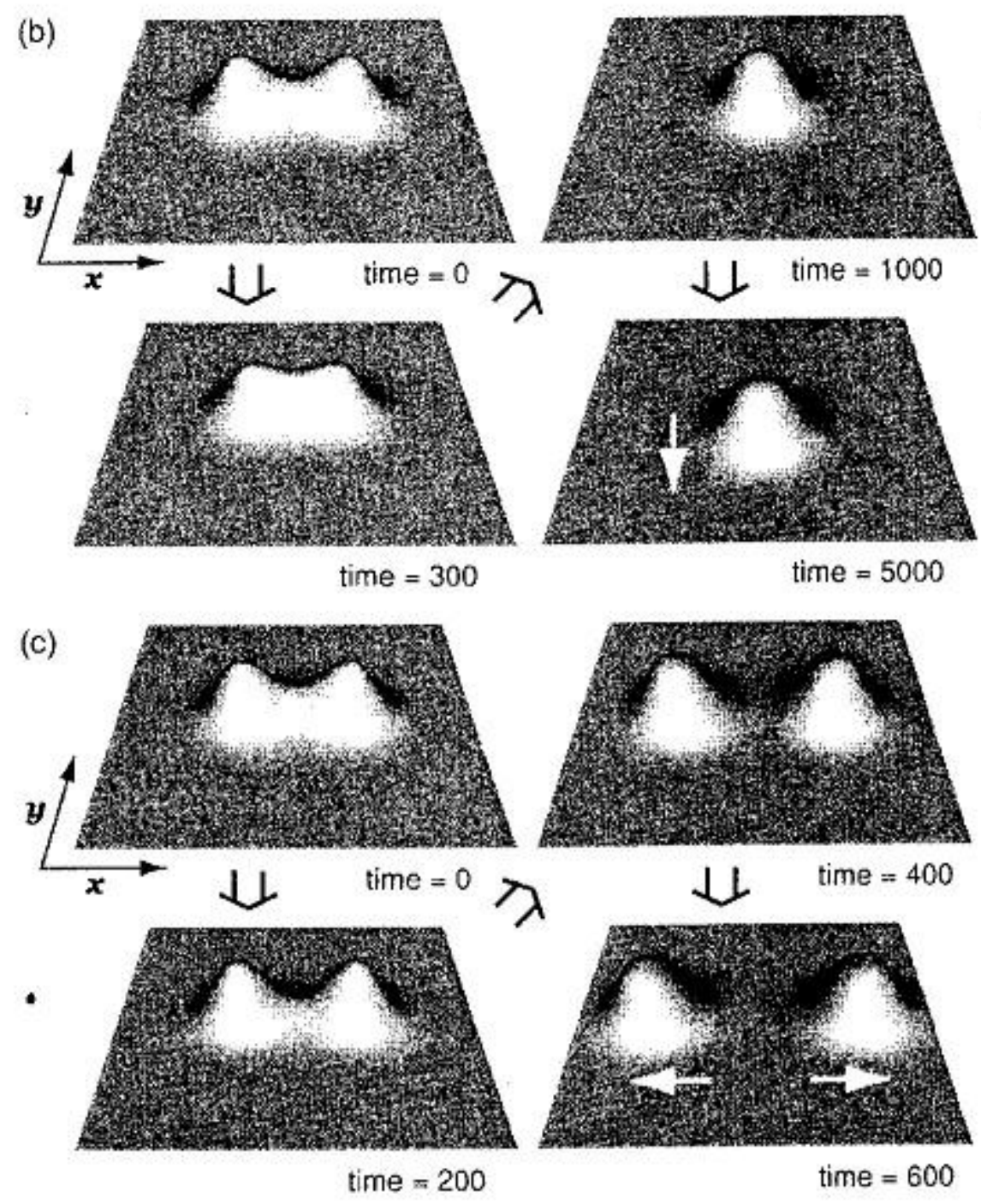

FIG. 7. (a) The peanut scattor at $\left(f_{1}, \tau\right)=(0.0625,63.777)$ (i.e., before the Hopf bifurcation) has only one unstable eigenvalue $\lambda_{1}=0.0141$ besides the drift instabilities. The associated eigenfunction $\Psi_{4}$ has flip symmetry with respect to the $x$ - and $y$-axes as in (a) with the cross section along the $x$-axis (right). Responses of the peanut scattor by adding a small perturbation in the direction of $\Psi_{1}$. (b) [respectively, (c)] positive (respectively, negative) perturbation. These are consistent with the outputs of Fig. 5.

sponding to the parameters on both sides behave in the following way. For FD side, it first approaches a peanut shape, then fuses into a small disk pattern, grows up to the large disk, stays there for certain time, and finally drifts away [see Fig. 8(b)]. On the other hand, for the AN side, after fusing into a small disk, it decays and annihilates [see Fig. 8(a)]. There appears a new scattor "small disk" in these processes, in fact it can be detected as in Fig. 8(c) and has codim 3 at this parameter. Like a large disk scattor, two of them are drifting instabilities and the remaining positive real one is responsible for the output, namely its unstable manifold goes to either the large disk or the homogeneous state (annihilation) as in Fig. 9. Note that the associated eigenfunction $\Phi_{1}$ in Fig. 9(a) also has flip symmetry like $\Psi_{1}$ in Fig. 7(a). The overall collision process can be understood clearly once we notice the two-step deformation, i.e., peanut shape $\rightarrow$ small disk, then small disk $\rightarrow$ large disk or small disk $\rightarrow$ annihilation. 
(a)
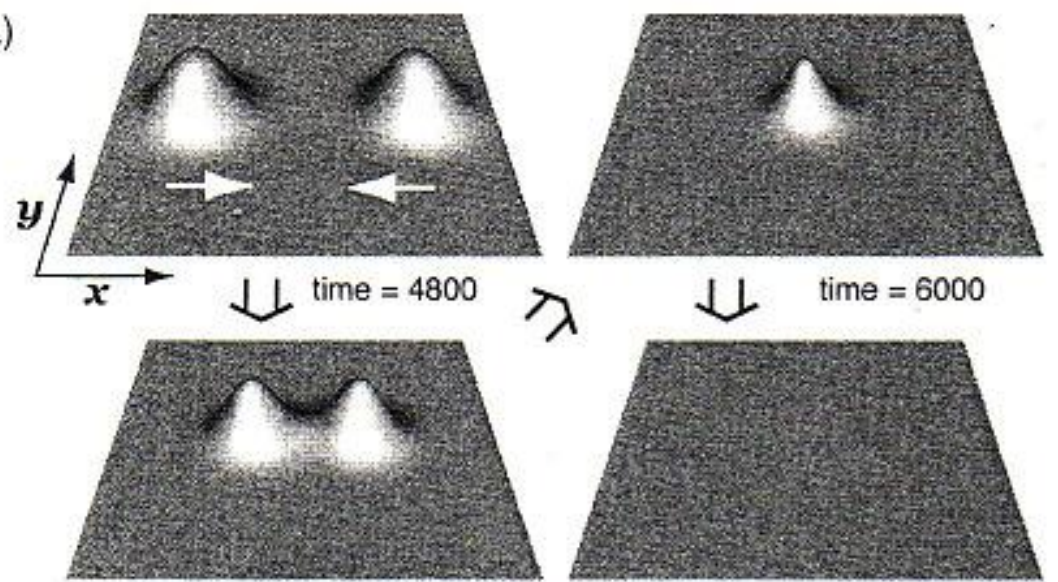

time $=5500$

(b)

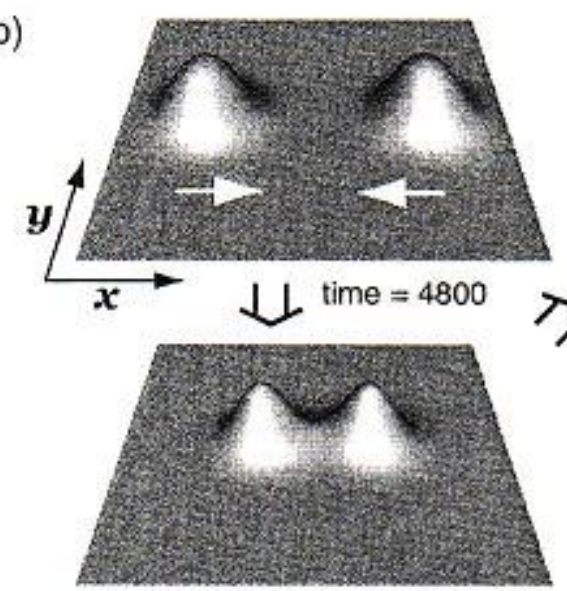
time $=5500$

(c)
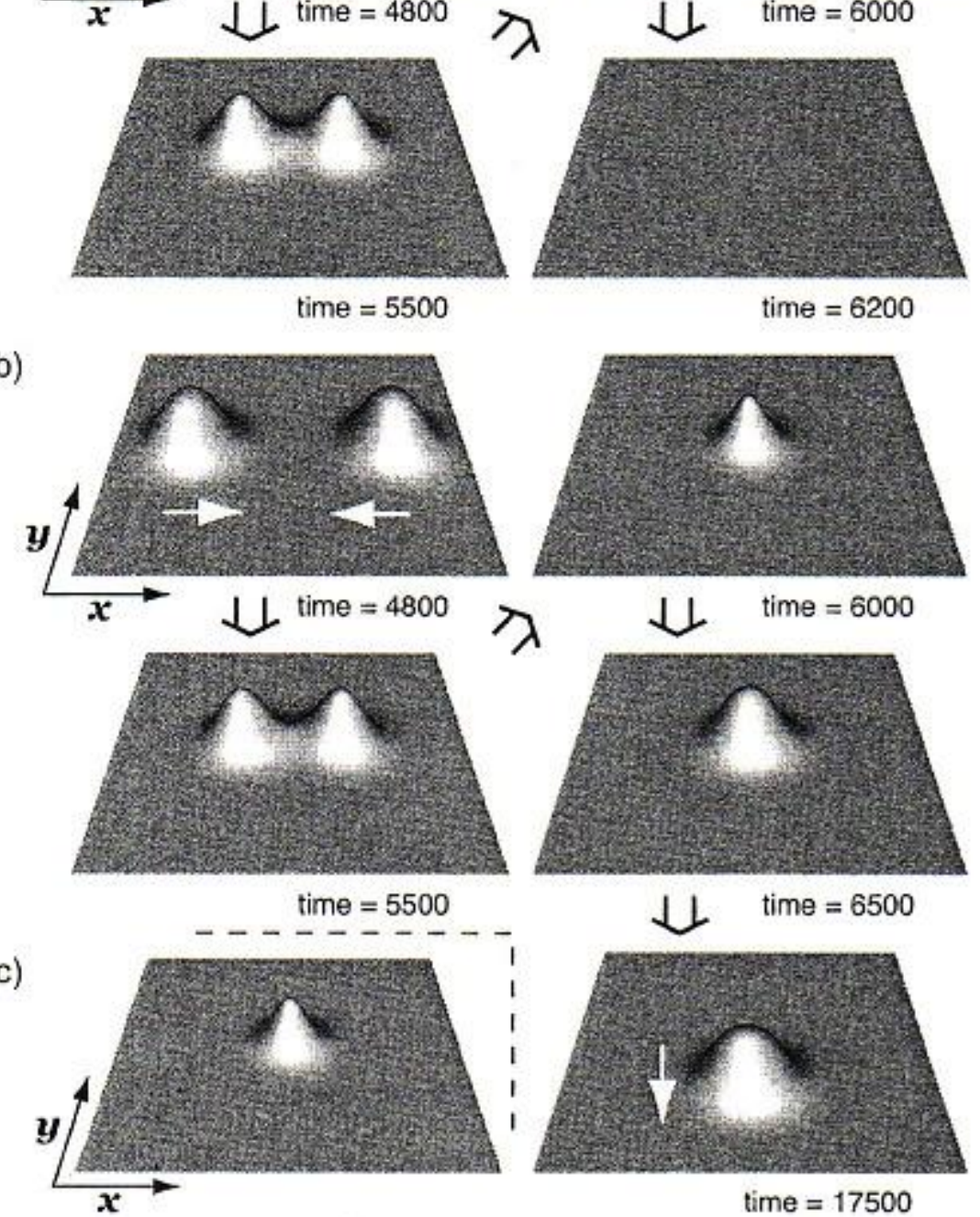

time $=6200$

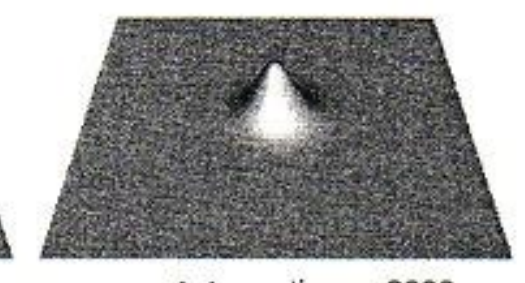

U time $=6000$

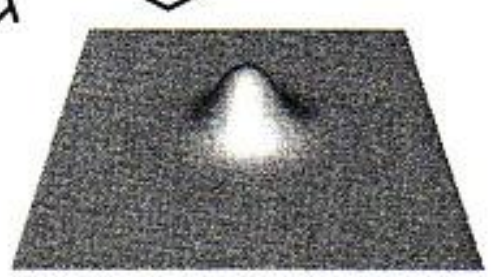

$\mathrm{me}=17500$

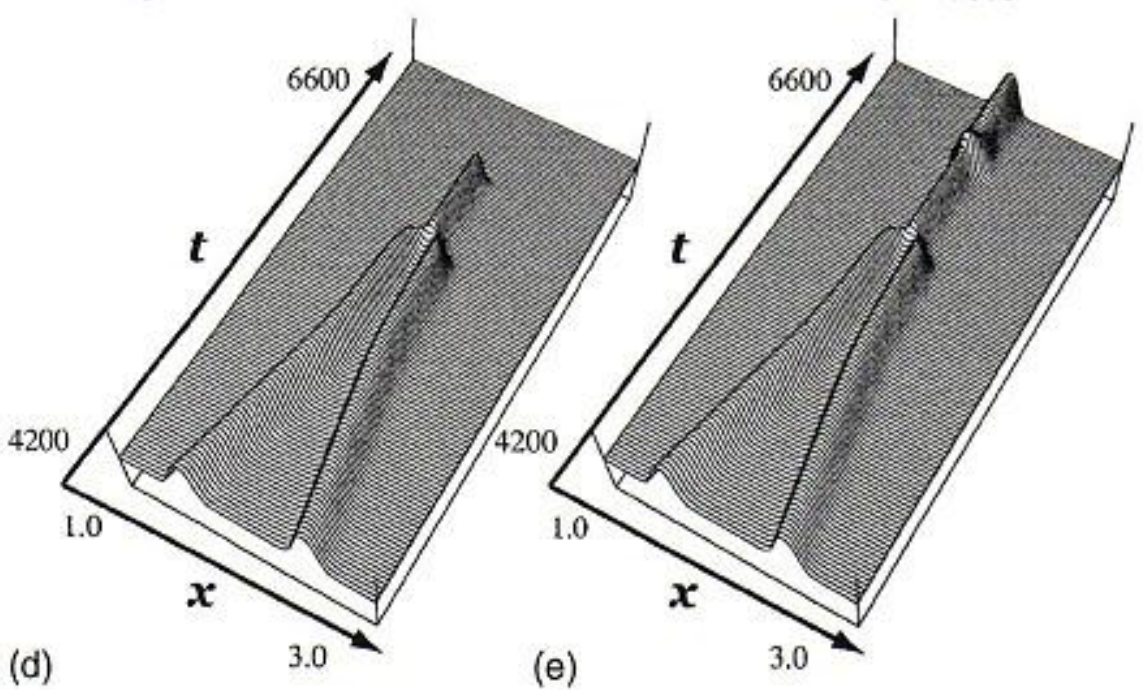

(e)

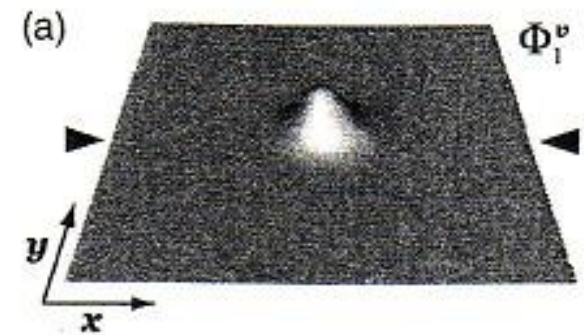

(b)
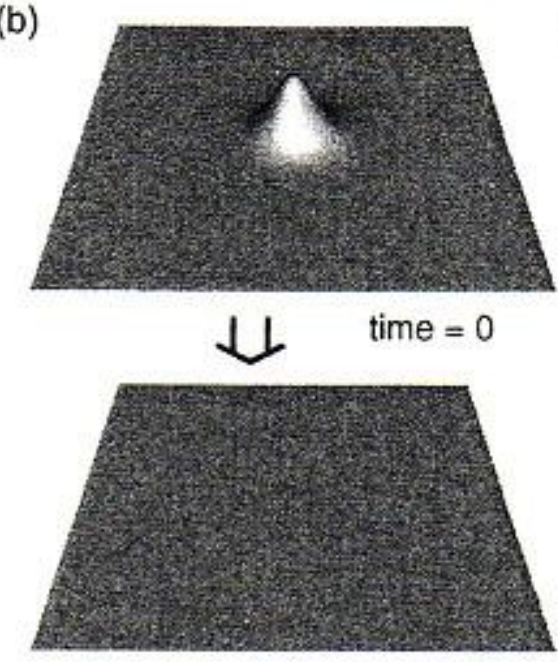

time $=200$

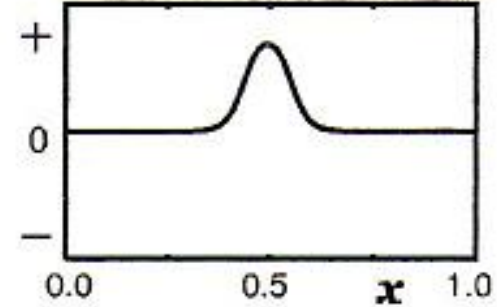

(c)
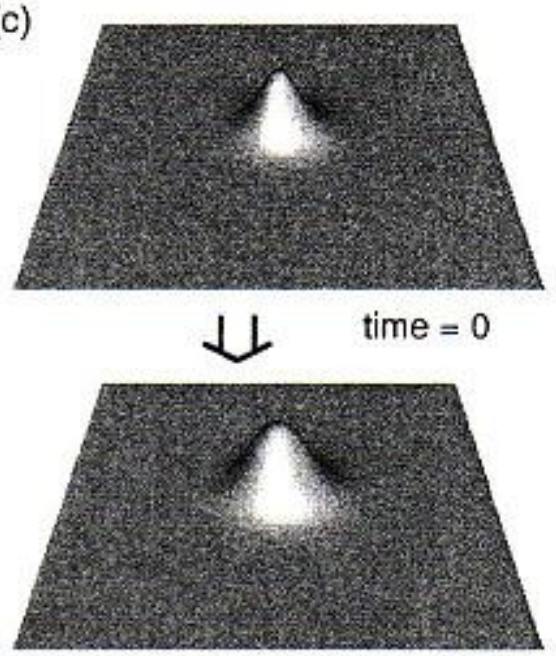

time $=800$

FIG. 9. (a) The unstable symmetric eigenfunction $\Phi_{1}$ of small disk solution at $\left(f_{1}, \tau\right) \approx(0.064578,90.0)$ with the profile along the $x$-axis (right). The eigenvalue is given to $\lambda_{1}=0.0646$. Response of the small disk scattor by a small perturbation in the direction of $\Phi_{1}$ (b) [respectively, (c)] negative (respectively, positive) perturbation, which are consistent with the outputs in Fig. 8.

Magnifying the TJ region, it turns out that the tip of the AN region undulates as $\tau$ changes, which may be caused by the Hopf instability of the peanut scattor discussed in the next section.

\section{GLOBAL NETWORK AMONG SCATTORS}

As observed in the preceding sections, a hidden saddle called the scattor plays a role like a traffic controller of the orbits depending on the parameters, however it is still unclear that how the annihilation regime emerges and the inputoutput relation changes from I to II as $f_{1}$ is increased. In this section we discuss this issue from the viewpoint of global interrelation among the scattors and by careful numerics for the behaviors of unstable manifolds of them. The scenario for the transition of input-output relation from I (FD-RE) to II (FD-AN) is as follows. When two spots approach, they first form a profile close to that of the peanut scattor, therefore the fate of the associated orbit is most probably controlled by the largest eigenvalue of Re parts of the spectrum around the peanut scattor. It turns out that the largest eigenvalue is switched from the real one to the complex pair as $f_{1}$ is increased (see Fig. 10), therefore the destination along the unstable manifold associated with the most dangerous one is also changed from large disk to small disk. The small disk has a 1D unstable manifold besides drift eigenvalues and one of the destinations is the homogeneous state (i.e., annihilation). The above crossover point is located at $f_{1} \approx 0.06421$ (slightly larger than TJ) at which the annihilation regime is clearly visible. The orbit is basically driven by the manner of connection among scattors and its switching causes the change of input-output relation as depicted in Fig. 11. 


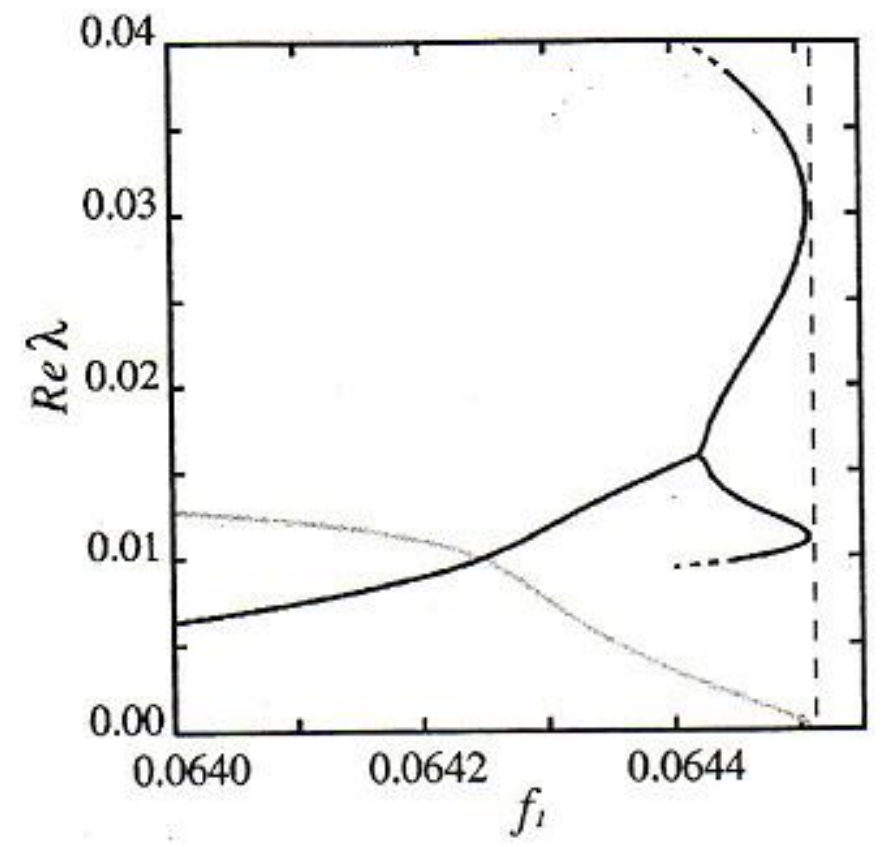

FIG. 10. $f_{t}$ dependence of the eigenvalues of the symmetric unstable mode for the large peanut solution for $\tau \approx 76.644$. The solid and gray lines indicate the values of the mode emanating from Hopf and symmetry-breaking bifurcations, respectively. The crossover point is located at $f_{1} \approx 0.06421$.

\section{A. Heteroclinic connection among scattors}

Recalling the RE-FD transition I in Fig. 3, the heteroclinic connection between the peanut scattor and the large disk forms a backbone for scattering dynamics. The orbits are sorted out like Fig. 12 depending on parameters.

On the other hand, for the FD-AN transition II in Fig. 3, a small disk emerges as a new scattor and becomes a part of the two-step deformation at collision, namely peanut $\rightarrow$ small disk $\rightarrow$ large disk or homogeneous state. It was
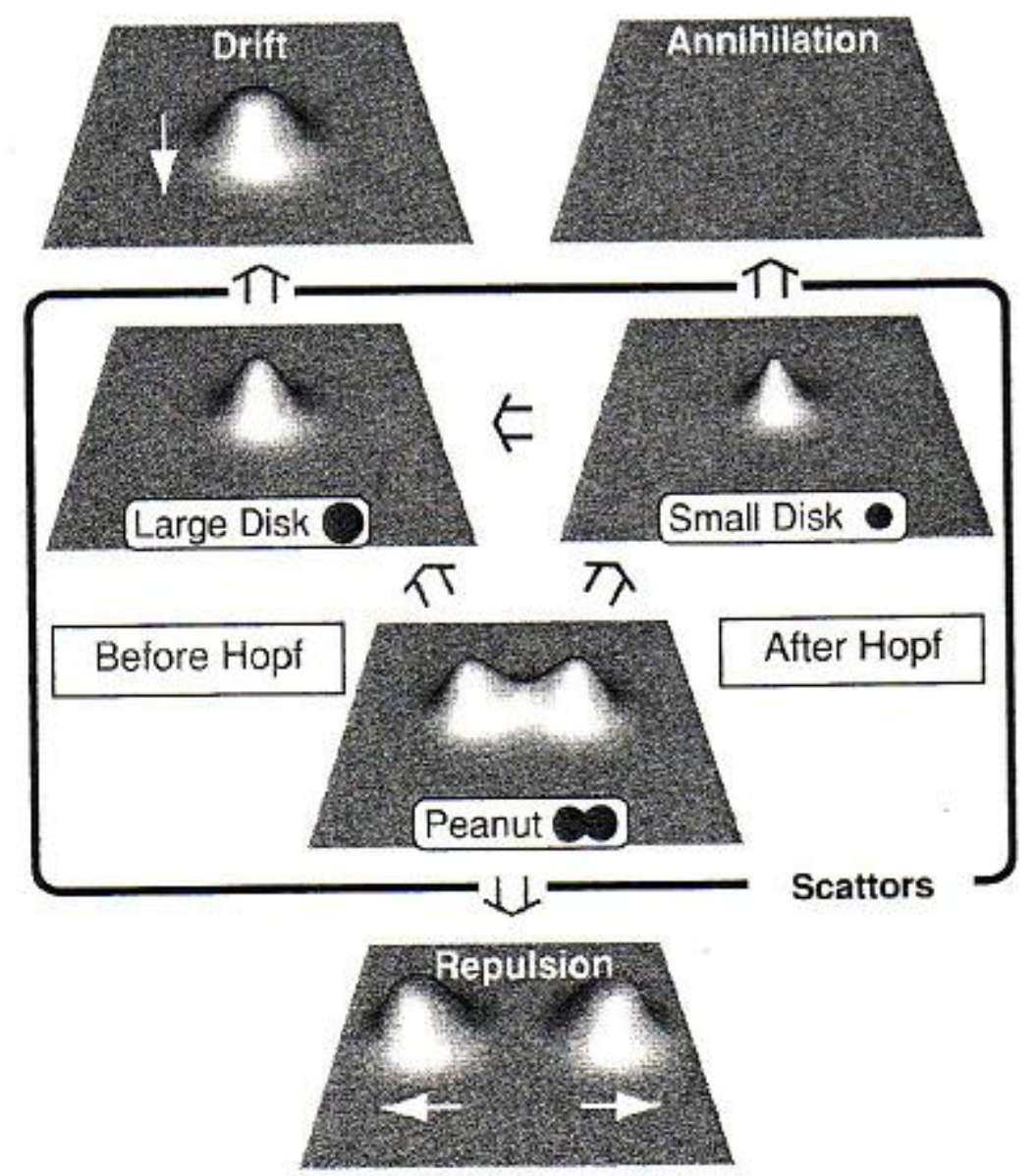

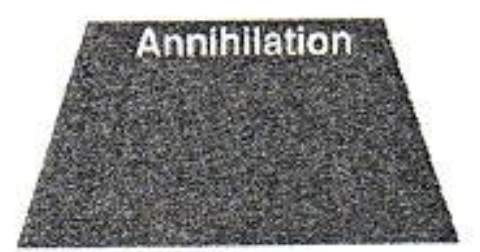

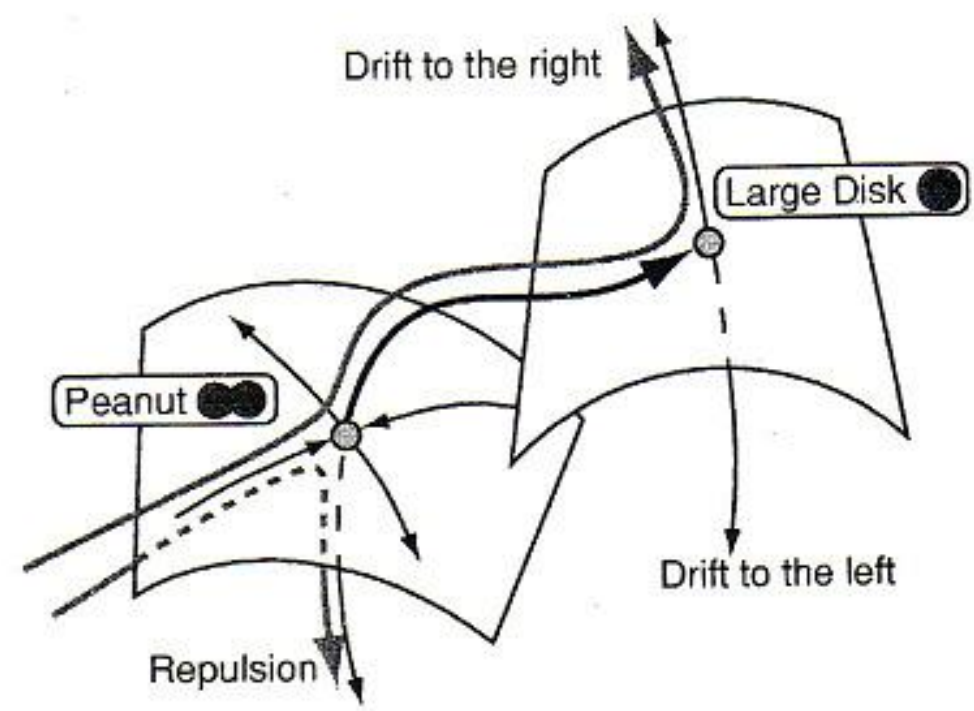

FIG. 12. Schematic diagram of the heteroclinic connection from peanut scattor to large disk scattor.

confirmed as in Fig. 9 that the unstable manifold of the small disk goes to either the large disk or the homogeneous state.

The aim of this section is to clarify the underlying structure controlling the above two transitions, especially how the annihilation regime emerges through the triple junction $\mathrm{TJ}$. In order to study the precise behaviors near TJ, we take $f_{1}$ $=0.0640$ for definiteness. In the neighborhood of this parameter regime, annihilation occurs typically in such a way that the orbit approaches the peanut shape, then the middle part of it starts to oscillate, and finally disappears. This suggests that some instability occurs for the peanut scattor as $f_{1}$ is increased as RE-FD regime. Moreover if a new instability really occurs, then we should study its implication, namely what we must do is the following:

(1) Study the spectral behavior around the peanut scattor along the $f_{1}$ axis.

(2) Search for the destination associated with the new instability.

A key information for the candidates comes from the global bifurcation diagram for scattors obtained in Sec. IV, here we show it in a superposed form with the phase diagram

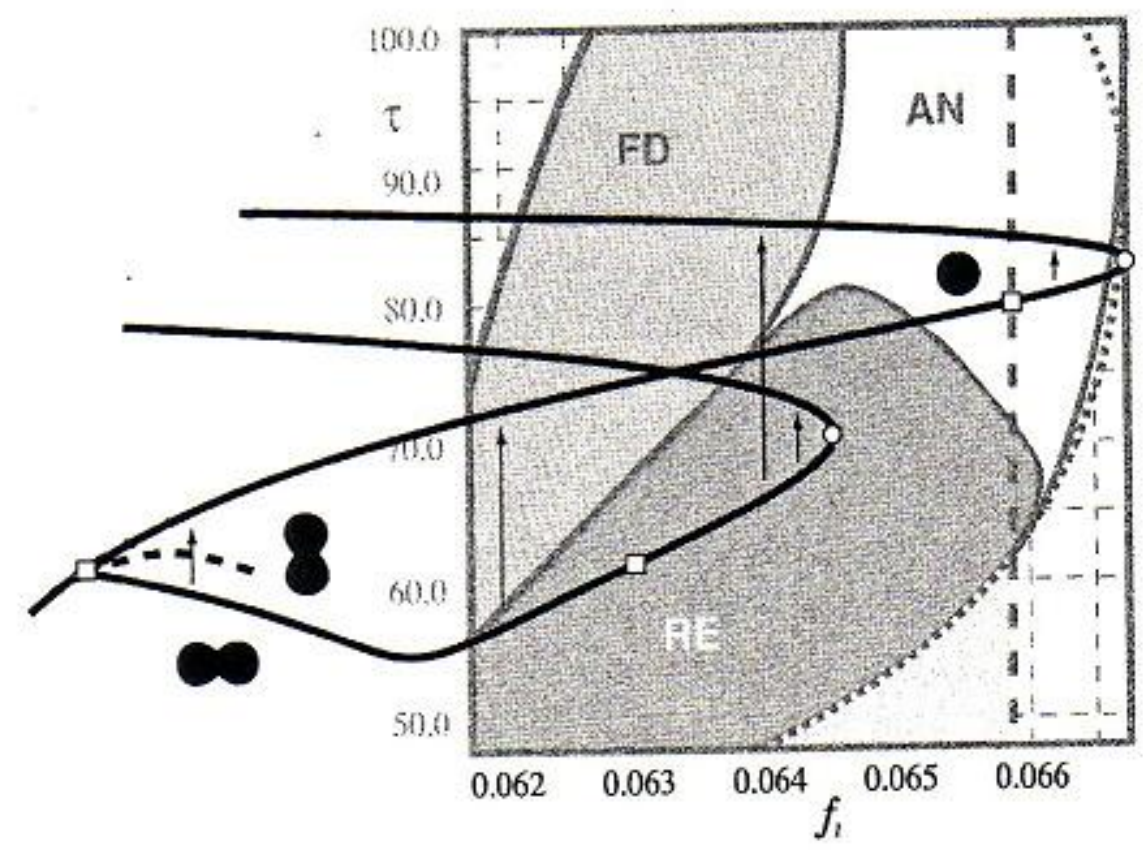

FIG. 13. Superposition of the $\left(f_{1}, \tau\right)$ phase diagram (Fig. 3) with the $f_{1}$ bifurcation diagram (Fig. 6).
FIG. 11. Schematic picture for the network of scattors. Three scattors are peanut scattor undergoes Hopf bifurcation. Small disk scattor is responsible for the occurrence of annihilation.

\footnotetext{
tre
}


(a)

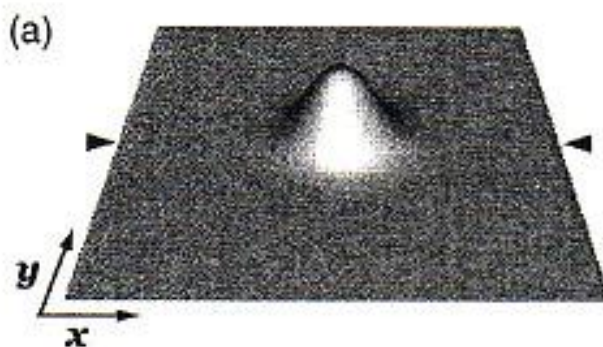

(b)

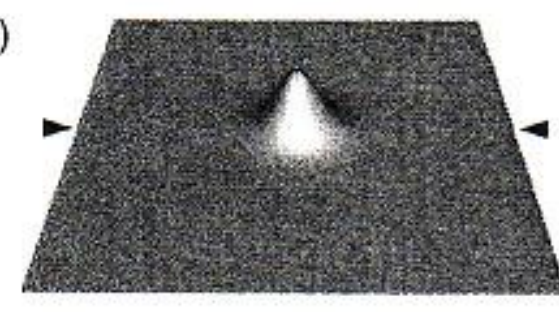

(c)

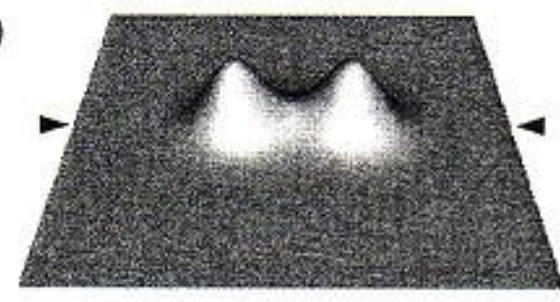

(d)

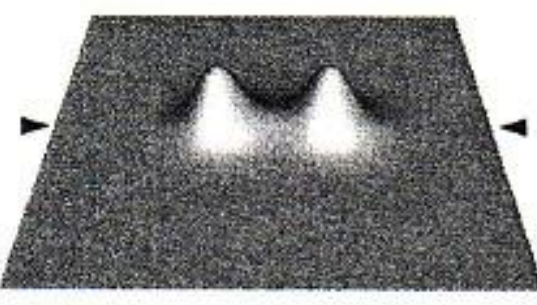

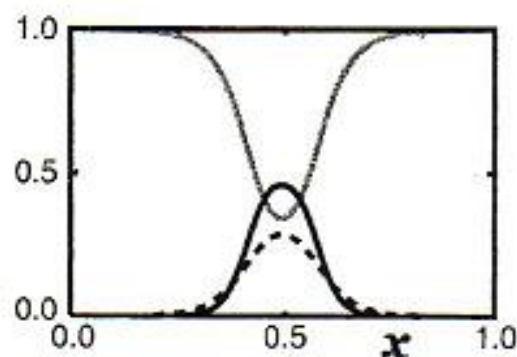
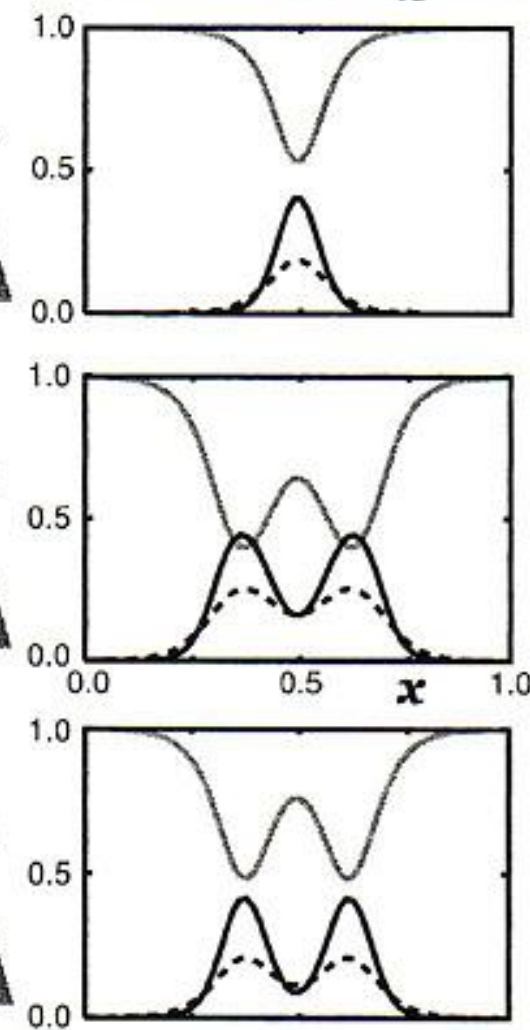

FIG. 14. (a) Profiles of scattors at $f_{1}=0.0640$. (a) Large disk solution at the lower branch, (b) small disk solution at the upper branch, (c) large peanut solution at the lower branch, (d) small peanut solution at the upper branch, only the $v$ component is shown here and the profile along the $x$-axis is depicted on the right-hand side. The gray, solid, and broken lines indicate $u$, $v$, and $w$ components, respectively.

as in Fig. 13. Large and small disks are connected via a saddle-node point and the branch of peanut scattor emanates from the branch of large disk via a symmetry-breaking bifurcation. The peanut branch also turns back via a saddle-node point. It should be noted that there occurs a Hopf bifurcation on the peanut branch around $f_{1} \approx 0.0630$ and the triple junction $\mathrm{TJ}$ appears slightly before $f_{1} \approx 0.0640$ and annihilation is clearly visible at $f_{1}=0.0642$. There are four steady scattors at $f_{1}=0.0640$ depicted in Fig. 14. We shall discuss in the next section how the interrelation among them is changed as $f_{1}$ is increased.

\section{B. Destabilization of peanut scattor implies switching of interconnections among scattors}

First we compute numerically the spectrum of the peanut scattor and its $f_{1}$ dependency. There are three dangerous eigenvalues as depicted in Fig. 10, a complex pair of eigenvalues (solid line) and one positive real eigenvalue (gray line). The complex pair originates in the Hopf bifurcation and the real one in the symmetry-breaking bifurcation from the large disk pattern. Recall that before Hopf bifurcation (i.e., for smaller $f_{1}$ ), only the real one is dangerous and it is sufficient to concentrate on the fate of the unstable manifold associated with it as in Fig. 5. The crossover point is located around $f_{1}=0.06421$, which coincides with the region where annihilation is clearly visible. In order to understand this switching, let us study the output from the peanut scattor for $f_{1}$
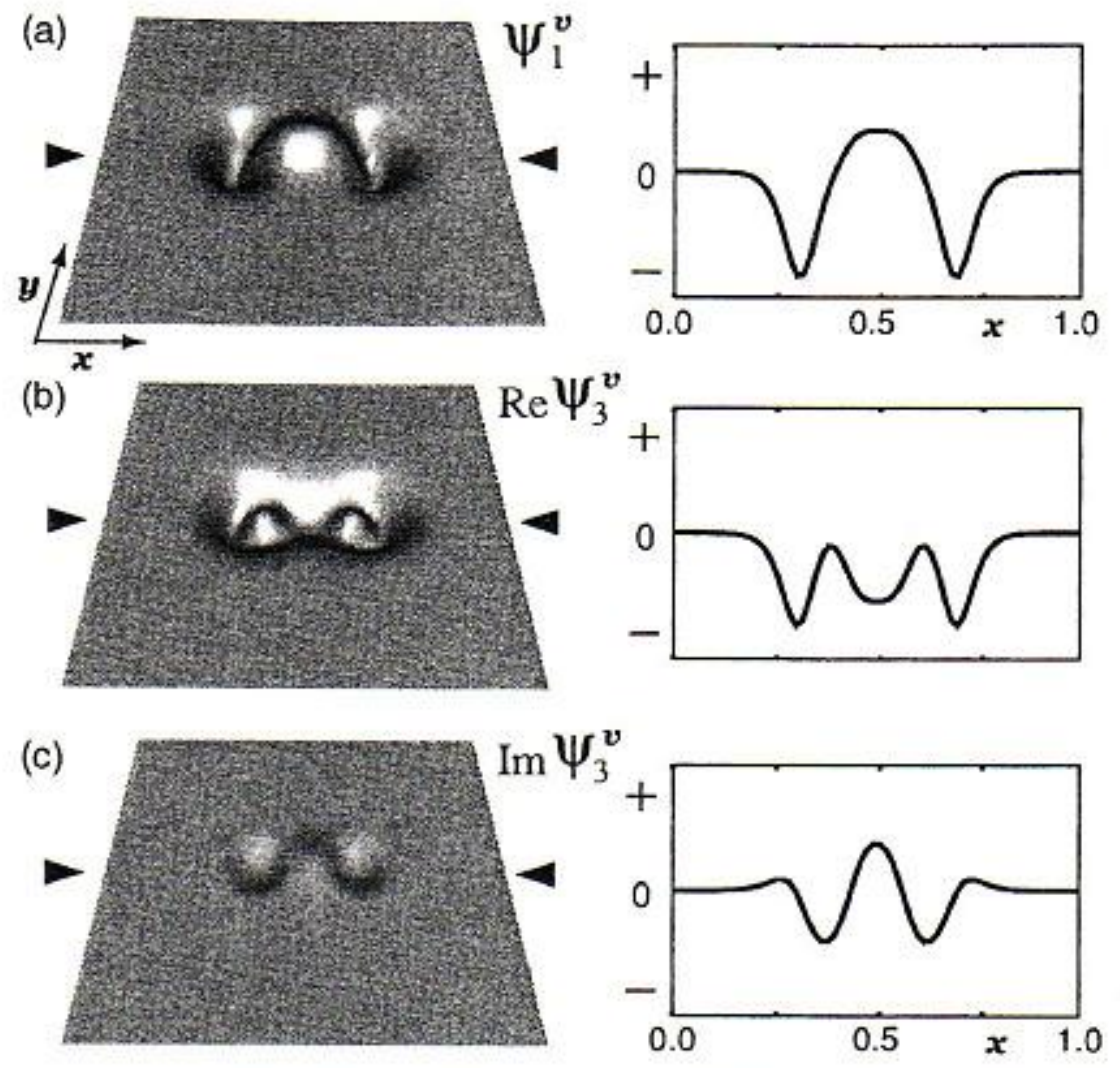

(d)

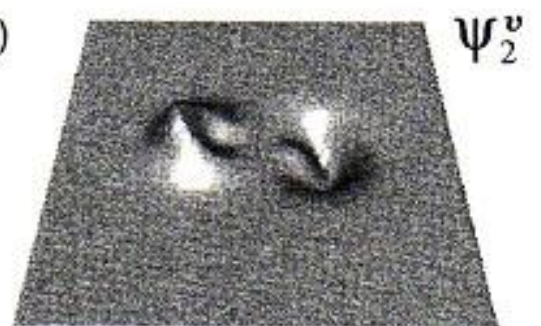

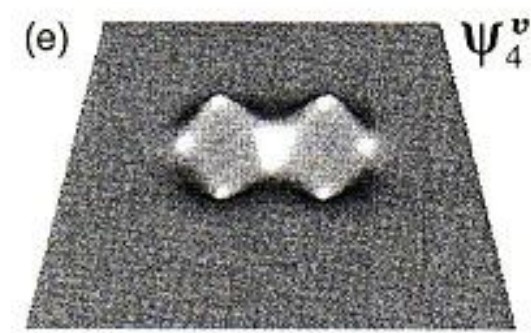

FIG. 15. The unstable eigenfunctions of large peanut solution at $\left(f_{1}, \tau\right)$ $\approx(0.0640,76.644)$ (i.e., after the Hopf bifurcation) with the profiles along the $x$-axis for the symmetric functions (a)-(c). The four eigenvalues are $\lambda_{1}=0.0127>\lambda_{2}=0.0107>\operatorname{Re}\left(\lambda_{3}=0.0063 \pm 0.0129 i\right)>\lambda_{4}=0.0008$. (d),(e) Two eigenfunctions associated with the drift instabilities.
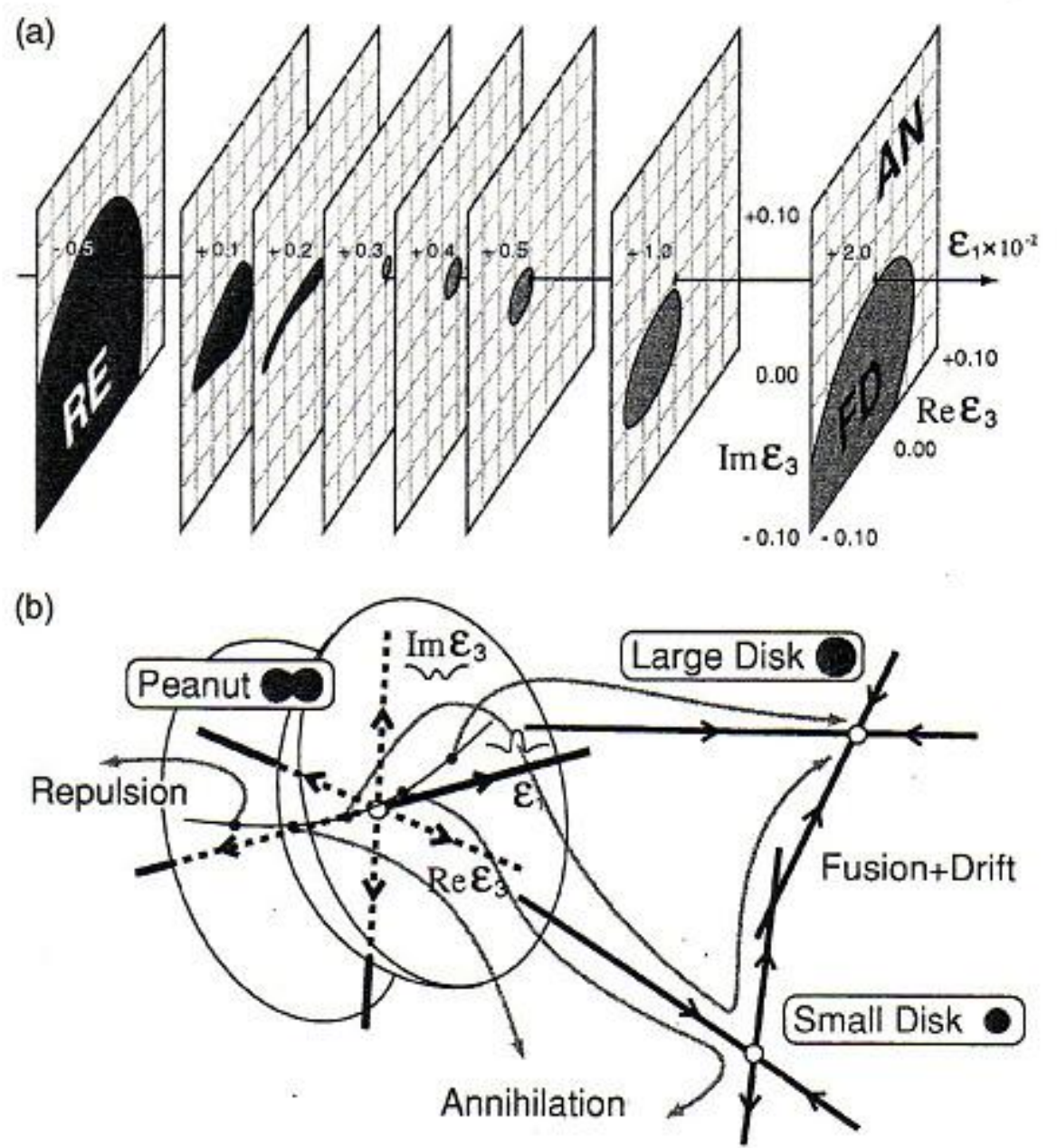

FIG. 16. (a) Phase diagram of responses of the peanut scattor by adding a small perturbation in the direction $\epsilon_{1} \Psi_{1}+\epsilon_{3} \Psi_{3}+\overline{\epsilon_{3} \Psi_{3}}$ when $\left(f_{1}, \tau\right)$ $\approx(0.0640,76.644)$. The $\left(\operatorname{Re} \epsilon_{3}, \operatorname{Im} \epsilon_{3}\right)$ slices are depicted along the $\epsilon_{1}$ axis. (b) Schematic picture of the connection orbit from peanut scattor to disk scattors. The solid and broken lines (respectively, gray lines) indicate the stable and unstable manifolds of the scattors (respectively, the typical orbits through the neighborhood of the peanut scattor). The destination of the orbits changes from RE to FD by increasing $\tau$. See the text for details. 
$=0.0640$ by adding a perturbation of linear combination of unstable directions. The linearized unstable eigenfunctions are computed as in Fig. 15, $\Psi_{1}$ (respectively, $\Psi_{3}$ ) corresponds to the largest real eigenvalue (respectively, the complex eigenvalue). Using this eigenform, we perturb the peanut scattor in the form of $\epsilon_{1} \Psi_{1}+\epsilon_{3} \Psi_{3}+$ c.c., the result of which is shown in Fig. 16. The horizontal line stands for the $\epsilon_{1}$ axis and each plane for the complex perturbation $\epsilon_{3}$. First of all, depending on the sign of $\epsilon_{1}$, the outputs are separated as fusion+drift (light-gray FD region for positive sign) and repulsion (dark-gray $\mathrm{RE}$ region for negative sign) up to the resolution $10^{-2}$ in a small neighborhood of the origin. This is a natural consequence of the fact that the symmetry-breaking real eigenvalue still dominates the dynamics at $f_{1}=0.0640$, however there appears an annihilation response (white AN region) for the rest part of the plane. The orbits starting from near the boundary of the FD-AN transition become very

(a)
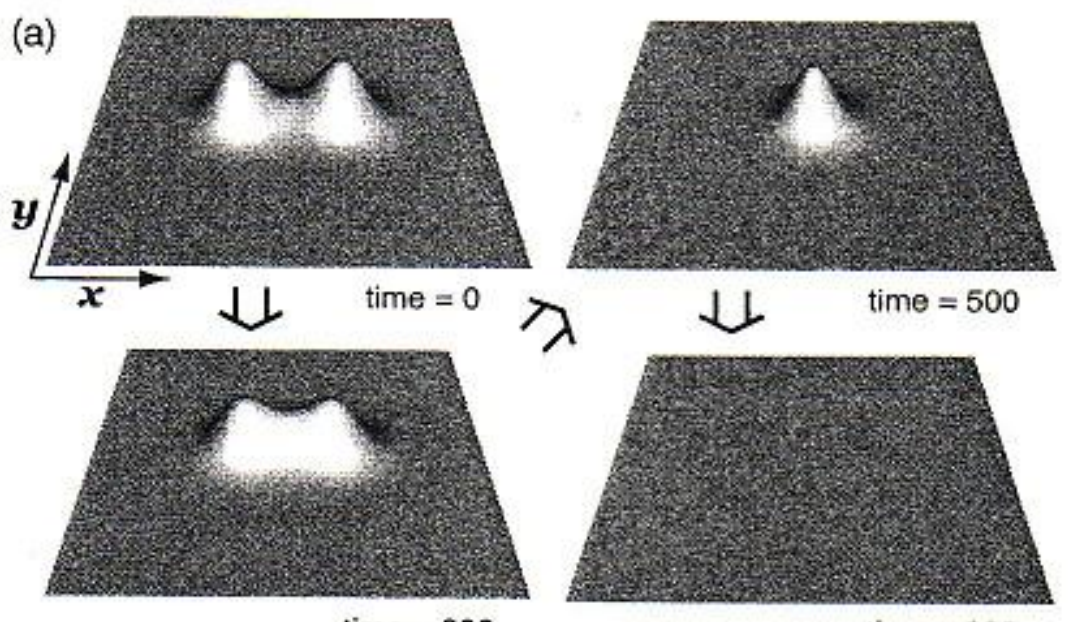

time $=300$

(b)
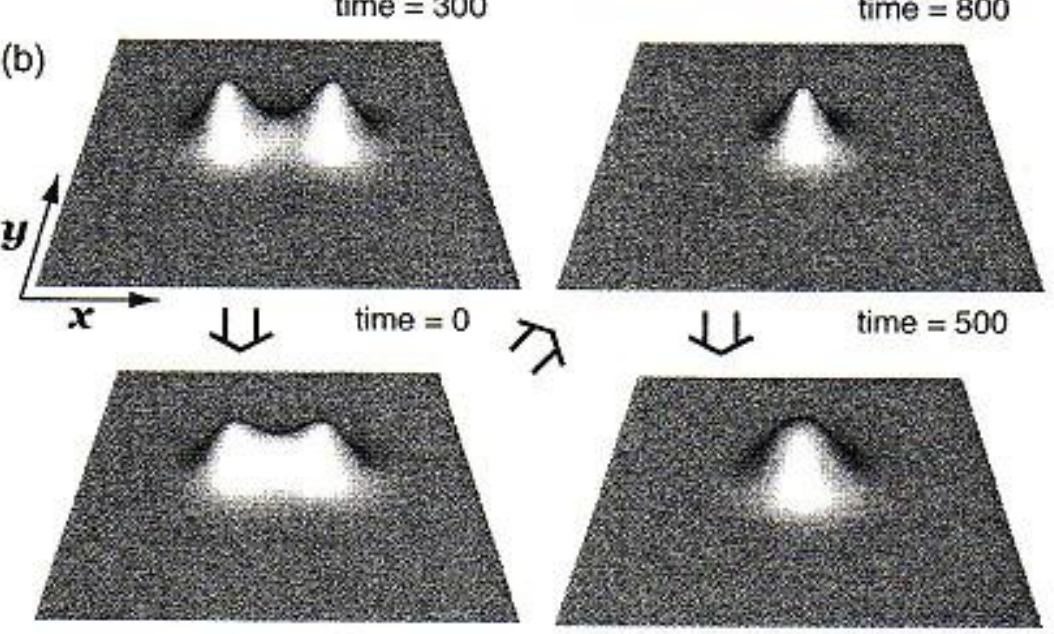

time $=300$

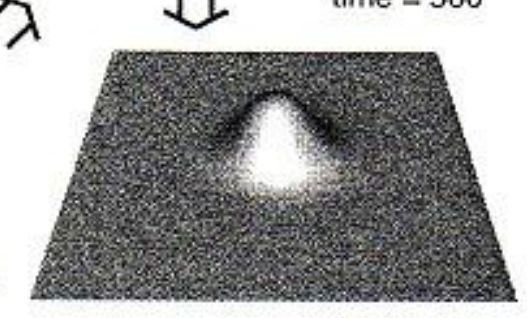

(c)

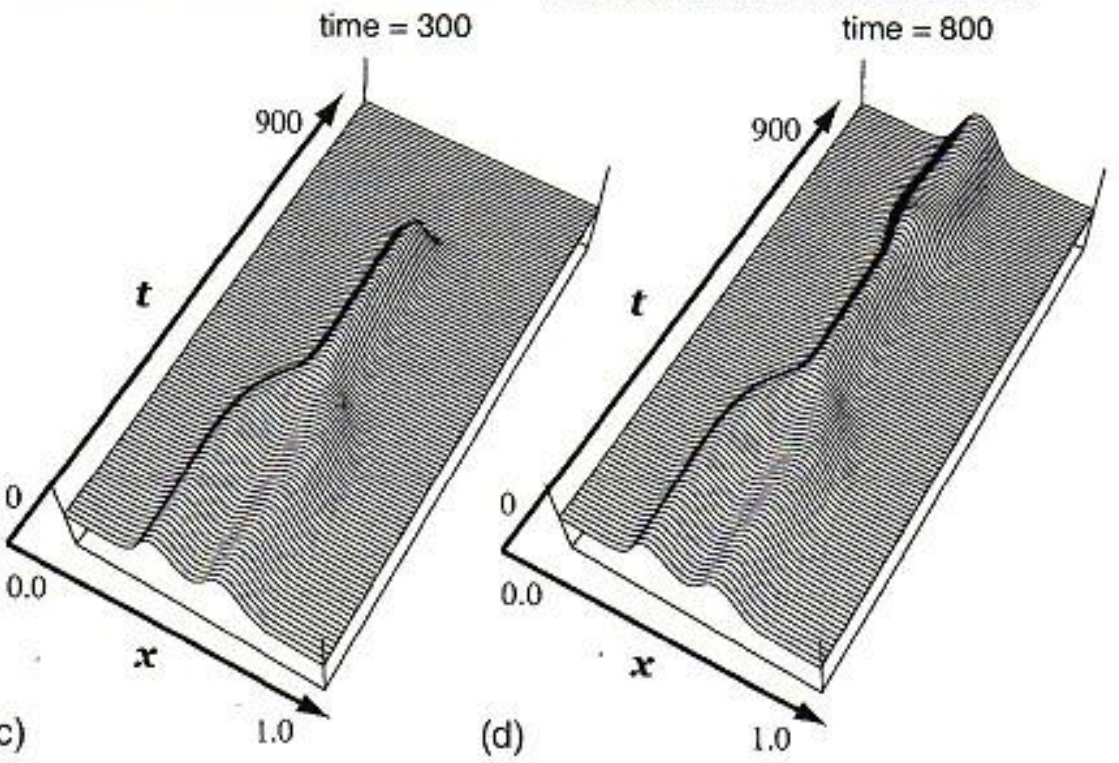

FIG. 17. Responses of the peanut scattor near FD-AN transition via small disk scattor for $\left(f_{1}, \tau\right) \approx(0.0640,76.667)$. The orbits with parameters taken along the FD-AN boundaries in Fig. 16(a) approach the small disk scattor closely. The associated time evolutions of the cross section along the $x$-axis are shown in (c) and (d). close to the small disk pattern, which causes annihilation. This is numerically confirmed for the peanut scattor located at $\left(f_{1}, \tau\right) \approx(0.0640,76.667)$ as shown in Fig. 17. These observations suggest that a new instability on the peanut branch leads to the new output AN. More precisely, annihilation occurs due to the emergence of new heteroclinicity from peanut scattor to small disk. Our discussions can be summarized in one schematic picture of Fig. 11, which shows the switching of main routes for orbits before and after Hopf bifurcation of peanut scattor.

\section{CONCLUDING REMARKS}

Scattering dynamics between two traveling spots and the transition manner of input-output relations of collision process are studied. We extend the idea developed in Refs. 22-24 to $2 \mathrm{D}$ traveling spots for the three-component system (1), namely we find three hidden saddles (called scattors), peanut, large and small disks which make a traffic control of orbits during the collision process and the orbital behaviors are guided by the stable and unstable manifolds of such scattors, and the output can be classified by looking at the outcome from the scattor by adding small perturbations. We highlight how the structural change of the network among scattors influences the input-output relations of the scattering dynamics. Such a change is typically triggered by a new instability of the scattor, which eventually leads to drastic change of output as parameters vary. We illustrate this with global behaviors of scattor's branch and precise numerical spectral analysis for (1). It is clarified that Hopf instability of the peanut scattor is responsible for the transition from the nonannihilation regime to the annihilation one.

Although we employ a specific three-component system (1), the approach presented here can be applicable for a wider class of reaction diffusion systems, in particular, the concept of scattor and its role for scattering dynamics seem to be universal, in fact the following three-component system (3), which was proposed as a qualitative model of a gas discharge system, ${ }^{32}$ has similar scattors with the same function (see Ref. 23 for details),

$$
\begin{aligned}
& u_{t}=D_{u} \Delta u+f(u)-\kappa_{3} v-\kappa_{4} w+\kappa_{1}, \\
& \tau v_{t}=D_{v} \Delta v+u-v, \\
& \theta w_{t}=D_{w} \Delta w+u-w,
\end{aligned}
$$

where $f(u)=2 u-u^{3}$. Strong collision is a very singular event for traveling spots, therefore scattors emerging at the transition point of input-output relations may turn out to be one of the universal objects independent of model systems.

\section{ACKNOWLEDGMENTS}

This work was partially supported by the Grant-in-Aid for Scientific Research (A)16204008 and Core Research for Evolutional Science and Technology (CREST), Japan Science and Technology Agency (JST):Creation of Novel Nanomaterial/system Synthesized by Self-organization for Medical Use. 
'P. de. Kepper, J. J. Perraud, B. Rudovics, and E. Dulos, Int. J. Bifurcation Chaos Appl. Sci. Eng. 4, 1215 (1994).

${ }^{2}$ K. J. Lee, W. D. McCormick, J. E. Pearson, and H. L. Swinney, Nature (London) 369, 215 (1994).

${ }^{3}$ V. Petrov, S. K. Scott and K. Showalter, Philos. Trans. R. Soc. London, Ser. A 347, 631 (1994).

${ }^{4}$ J. E. Pearson, Science 216, 189 (1993).

${ }^{5}$ Y. Astrov and H.-G. Purwin, Phys. Lett. A 283, 349 (2001).

${ }^{6}$ M. Bode, A. W. Liehr, C. P. Schenk, and H.-G. Purwins, Physica D 161 , 45 (2002).

${ }^{7}$ S. Nasuno, Chaos 13, 1010 (2003).

${ }^{8}$ M. Bär, M. Eiswirth, H.-H. Rotermund, and G. Ertl, Phys. Rev. Lett. 69 , 945 (1992).

${ }^{9}$ M. G. Zimmermann et al., Physica D 110, 92 (1997).

${ }^{10}$ A. von Oertzen, A. S. Mikhailov, H. H. Rotermund, and G. Ertl, J. Phys. Chem. B 102, 4966 (1998),

${ }^{11}$ S.-I. Ei, M. Mimura, and M. Nagayama, Physica D 165, 176 (2002).

${ }^{12}$ M. Mimura and M. Nagayama, Chaos 7, 817 (1997).

${ }^{13}$ T. Ohta, J. Kiyose, and M. Mimura, J. Phys. Soc. Jpn. 66, 1551 (1997).

${ }^{14}$ T. Ohta, Physica D 151, 61 (2001).

${ }^{15} \mathrm{H}$. Meinhardt, The Algorithmic Beauty of Sea Shells (Springer-Verlag, Berlin, 1999).

${ }^{16}$ K. Krischer and A. Mikhailov, Phys. Rev. Lett. 73, 3165 (1994).

${ }^{17}$ S. Kawaguchi and M. Mimura, SIAM J. Appl. Math. 59, 920 (1999).
${ }^{18}$ Y. Nishiura and D. Ueyama, Physica D 150, 137 (2001).

${ }^{19}$ Y. Hayase and T. Ohta, Phys. Rev. E 62, 5998 (2000).

${ }^{20}$ Y. Nishiura and D. Ueyama, Physica D 130, 73 (1999).

${ }^{21}$ Y. Nishiura, Far-from-Equilibrium Dynamics, Translations of Mathematical Monographs Vol. 209 (American Mathematical Society, Providence, RI, 2002).

${ }^{22}$ Y. Nishiura, T. Teramoto, and K.-I. Ueda, Phys. Rev. E 67, 056210 (2003).

${ }^{23}$ Y. Nishiura, T. Teramoto, and K.-I. Ueda, Chaos 13, 962 (2003).

${ }^{24}$ T. Teramoto, K.-I. Ueda, and Y. Nishiura. Phys. Rev. E 69, 056224 (2004).

${ }^{25}$ M. Argentina, P. Coullet, and L. Mahadevan, Phys. Rev. Lett. 79, 2803 (1997).

${ }^{26}$ M. Argentina, P. Coullet, and V. Krinsky, J. Theor. Biol. 205, 47 (2000).

${ }^{27}$ M. van Hecke and M. Howard, Phys. Rev. Lett. 86, 2018 (2001).

${ }^{28}$ M. Argentina, O. Rudzick, and M. G. Velarde, Chaos 14, 777 (2004).

${ }^{29}$ C. P. Schenk, M. Or-Guil, M. Bode, and H.-G. Purwins, Phys. Rev, Lett. 78, 3781 (1997).

${ }^{30} \mathrm{Y}$. Nishiura, T. Teramoto, and K.-I. Ueda (preprint).

${ }^{31}$ E. J. Doedel, A. R. Champneys, T. F. Fairgrieve, Y. A. Kuznetsov, B. Sandstede, and X. Wang, AUTO97, http://cmul.cs.concordia.ca/auto (1997).

${ }^{32} \mathrm{H},-\mathrm{G}$. Purwins, Y. Astrov, and I. Brauer, in The Fifth Experimental Chaos Conference, edited by M. Ding, W. L. Ditto, L. M. Pecora, and M. L. Spano (World Scientific, Singapore, 2001), p. 3. 